\title{
Early high-dose vitamin $C$ in post-cardiac arrest syndrome (VITaCCA): study protocol for a randomized, double-blind, multi- center, placebo-controlled trial
}

Sander Rozemeijer ${ }^{1 *}$ (D), Harm-Jan de Grooth ${ }^{1,2}$, Paul W. G. Elbers ${ }^{1}$, Armand R. J. Girbes ${ }^{1}$, Corstiaan A. den Uil ${ }^{3}$, Eric A. Dubois ${ }^{4,5}$, Evert-Jan Wils ${ }^{6}$, Thijs C. D. Rettig ${ }^{7}$, Arthur R. H. van Zanten ${ }^{8,9}$, Roel Vink ${ }^{10}$, Bas van den Bogaard ${ }^{11}$, Rob J. Bosman ${ }^{11}$, Heleen M. Oudemans-van Straaten ${ }^{1}$ and Angélique M. E. de Man ${ }^{1}$

\begin{abstract}
Background: High-dose intravenous vitamin $C$ directly scavenges and decreases the production of harmful reactive oxygen species (ROS) generated during ischemia/reperfusion after a cardiac arrest. The aim of this study is to investigate whether short-term treatment with a supplementary or very high-dose intravenous vitamin $C$ reduces organ failure in post-cardiac arrest patients.

Methods: This is a double-blind, multi-center, randomized placebo-controlled trial conducted in 7 intensive care units (ICUs) in The Netherlands. A total of 270 patients with cardiac arrest and return of spontaneous circulation will be randomly assigned to three groups of 90 patients (1:1:1 ratio, stratified by site and age). Patients will intravenously receive a placebo, a supplementation dose of $3 \mathrm{~g}$ of vitamin $\mathrm{C}$ or a pharmacological dose of $10 \mathrm{~g}$ of vitamin ( per day for $96 \mathrm{~h}$. The primary endpoint is organ failure at $96 \mathrm{~h}$ as measured by the ResuscitationSequential Organ Failure Assessment (R-SOFA) score at $96 \mathrm{~h}$ minus the baseline score (delta R-SOFA). Secondary endpoints are a neurological outcome, mortality, length of ICU and hospital stay, myocardial injury, vasopressor support, lung injury score, ventilator-free days, renal function, ICU-acquired weakness, delirium, oxidative stress parameters, and plasma vitamin C concentrations.
\end{abstract}

Discussion: Vitamin C supplementation is safe and preclinical studies have shown beneficial effects of high-dose IV vitamin C in cardiac arrest models. This is the first RCT to assess the clinical effect of intravenous vitamin C on organ dysfunction in critically ill patients after cardiac arrest.

Trial registration: ClinicalTrials.gov NCT03509662. Registered on April 26, 2018. https:/clinicaltrials.gov/ct2/show/ NCT03509662European Clinical Trials Database (EudraCT): 2017-004318-25. Registered on June 8, 2018. https://www. clinicaltrialsregister.eu/ctr-search/trial/2017-004318-25/NL

\footnotetext{
* Correspondence: s.rozemeijer@amsterdamumc.nl

'Department of Intensive Care Medicine, Research VUmc Intensive Care (REVIVE), Amsterdam Cardiovascular Science (ACS), Amsterdam Infection and Immunity Institute (AI\&ll), Amsterdam Medical Data Science (AMDS),

Amsterdam UMC, Location VUmc, Vrije Universiteit Amsterdam, De Boelelaan 1117, 1081 HV Amsterdam, The Netherlands

Full list of author information is available at the end of the article
}

(c) The Author(s). 2021 Open Access This article is licensed under a Creative Commons Attribution 4.0 International License, which permits use, sharing, adaptation, distribution and reproduction in any medium or format, as long as you give appropriate credit to the original author(s) and the source, provide a link to the Creative Commons licence, and indicate if changes were made. The images or other third party material in this article are included in the article's Creative Commons licence, unless indicated otherwise in a credit line to the material. If material is not included in the article's Creative Commons licence and your intended use is not permitted by statutory regulation or exceeds the permitted use, you will need to obtain permission directly from the copyright holder. To view a copy of this licence, visit http://creativecommons.org/licenses/by/4.0/ The Creative Commons Public Domain Dedication waiver (http://creativecommons.org/publicdomain/zero/1.0/) applies to the data made available in this article, unless otherwise stated in a credit line to the data. 
Keywords: Out-of-hospital cardiac arrest, Ischemia/reperfusion injury, Post-cardiac arrest syndrome, Cardiac arrest, Free radicals, Reactive oxygen species, Oxidative stress, Vitamin C, Ascorbic acid

\section{Background}

Intravenous treatment with high-dose vitamin $C$ may improve the clinical outcome of post-cardiac arrest patients with ROSC (return of spontaneous circulation), because reactive oxygen species (ROS), generated during the systemic ischemic-reperfusion response, contribute to organ damage and death $[1,2]$. Vitamin $\mathrm{C}$ directly scavenges free radicals, repairs oxidized scavengers such as glutathione, and reduces the production of ROS [3]. As a result, it may reduce ischemia/reperfusion injury. Due to the massive release of ROS, vitamin $\mathrm{C}$ stores become exhausted and vitamin $\mathrm{C}$ plasma concentrations decrease to deficiency levels within 3 days after a cardiac arrest [4-6]. Enteral supplementation of vitamin $C$ cannot restore plasma concentrations in critically ill patients due to limited absorption and acutely increased requirements [7-10]. Intravenous supplementation is therefore required to restore the deficiency [11-14] or to achieve higher plasma concentrations [12].

Beneficial effects of high-dose intravenous (IV) vitamin $\mathrm{C}$ on myocardial and cerebral ischemia-reperfusion injury have been reported in preclinical studies [2]. In clinical studies, IV administration of vitamin $\mathrm{C}$ reduced cardiac injury during percutaneous coronary intervention (PCI) [15] and reduced the incidence of atrial fibrillation following cardiac surgery [16]. The effects of IV vitamin $\mathrm{C}$ after cardiac arrest have been demonstrated in preclinical studies, but not in patients [11], and the optimal dose has not been determined yet.

In septic patients, a population also exposed to severe oxidative stress, three small studies and one larger randomized controlled trial have shown promising results. High-dose intravenous vitamin $\mathrm{C}$ alone [17, $18]$, or in combination with thiamine and hydrocortisone $[19,20]$, improved organ failure recovery, shock reversal, and survival. Results on organ failure improvement and vasopressor requirement in other recently published trials are not uniform [21-28]. Ongoing randomized controlled trials hopefully provide more conclusive answers [29].

Currently, the prognosis of cardiac arrest patients remains poor [30]. The most important therapeutic target in out-of-hospital cardiac arrest (OHCA) is shortening the time to ROSC (minimizing no flow and low flow state) by early chest compressions and defibrillation. Once the circulation has recovered, no effective therapy that diminishes the ischemia/reperfusion injury has been developed yet besides targeted temperature management [1].
Therefore, we want to study whether a short-term treatment with supplementary or high-dose intravenous vitamin $C$, when administered in the early phase of postcardiac arrest reperfusion, can limit organ damage.

\section{Methods \\ Study design}

The early high-dose vitamin $\mathrm{C}$ in post-cardiac arrest syndrome (VITaCCA) trial is a double-blind, multicenter, randomized placebo-controlled trial with a threearm comparative design. The study was approved by the ethics committee at Amsterdam UMC, location VUmc (VU University Medical Centre Protocol Record METC2018.120) and will be performed in adherence to the Declaration of Helsinki. The trial was prospectively registered at ClinicalTrials.gov on April 26, 2018, with identifier NCT03509662. The recruiting of patients started on October 7, 2019.

This clinical trial will be conducted in seven Dutch ICUs: Amsterdam UMC, Location VUmc (coordinating center), Gelderse Vallei Hospital, Franciscus Gasthuis \& Vlietland, Tergooi Hospital, Amphia Hospital, Erasmus MC and OLVG, location East.

\section{Population}

The study population consists of adult patients who suffered an out-of-hospital cardiac arrest. Patients must meet all of the inclusion criteria and none of the exclusion criteria in order to participate in this study (Table 1). Patients will be screened for eligibility by the attending physicians. A study flow chart is provided in Fig. 1.

\section{Study protocol Interventions}

As soon as possible after arrival at the Emergency Department, patients will be randomly allocated to one of three treatment groups. Group 1 will be treated with a placebo $(0.9 \% \mathrm{NaCl})$, group 2 with a twice-daily intravenous bolus of $1.5 \mathrm{~g}$ vitamin $\mathrm{C}$, and group 3 with a twice-daily intravenous bolus of $5 \mathrm{~g}$ vitamin $\mathrm{C}$ for 4 days or until ICU discharge. Ascorbic acid ampoules (100 $\mathrm{mg} / \mathrm{ml}$ ) from Centrafarm, Etten-Leur, the Netherlands, will be used. Placebo or vitamin $\mathrm{C}$ (diluted in $0.9 \% \mathrm{NaCl}$ ) is administered intravenously per $50 \mathrm{ml}$ and is infused in $15 \mathrm{~min}$, each $12 \mathrm{~h}$. The first dose of the study medication is aimed to be administered within $5 \mathrm{~h}$ after ROSC. A total of eight infusions will be given in 4 days. In addition, all patients will receive intravenous thiamine 
Table 1 Inclusion and exclusion criteria

\begin{tabular}{ll}
\hline Inclusion criteria & Exclusion criteria \\
\hline An out-of-hospital cardiac arrest with return of spontaneous circulation (ROSC); & Terminal renal insufficiency, i.e., receiving renal replacement \\
Ventricular fibrillation (VF) or ventricular tachycardia (VT) as first registered cardiac & therapy (RRT); \\
rhythm; & Known glucose 6-phosphate dehydrogenase deficiency (risk of \\
Glasgow Coma Scale (GCS) $\leq 8^{b}$. & hemolysis); \\
& $\begin{array}{l}\text { History of urolithiasis, oxalate nephropathy, or hemochromatosis; } \\
\text { Treatment limitations }\end{array}$
\end{tabular}

${ }^{a}$ If an automated external defibrillator advised to shock, then VF or VT was registered by the device.

bThe last GCS-score before the start of sedatives will be used.

${ }^{\mathrm{c}}$ The presence of treatment limitations will only be assessed at the moment of randomization.

$200 \mathrm{mg}$ every $12 \mathrm{~h}$ for 4 days or until ICU discharge to limit the oxalate production [19].

Further post-cardiac arrest care, including targeted temperature management (TTM), is in accordance with international guidelines [31] and local procedures [32]. Standard intravenous vitamin C supplementation is allowed for dosages up to $200 \mathrm{mg}$ a day. In case of clinical indication, higher dosages of thiamine are permitted. In case of irreversible coma, the procedure to withdraw life-sustaining treatment will take place according to Dutch guidelines [32].

\section{Randomization, treatment allocation, and blinding}

Patients will be randomized with the use of a computergenerated randomization list. The randomization will be stratified by site and age ( $\leq 66$ and $>66$ years) in randomized blocks of 6 . The patient will be randomized, and thereby automatically allocated to a treatment group, by an unblinded nurse or pharmacy assistant not involved in the direct care of the included patient. The study medication will be prepared by this unblinded health care worker in an amber-colored syringe that will be connected to an orange infusion line. A blinded nurse involved in the direct care of the included patient will administer the prepared study medication to the patient. The patient, physicians, and researchers involved are blinded for the allocated treatment during the entire study period. Unblinding will only take place in case of a suspected unexpected serious adverse reaction (SUSAR). Then, the administration of study medication will be stopped immediately.

\section{Deferred consent}

The ethics committee approved to start with study interventions before informed consent is obtained to avoid treatment delay. The legal representative will be informed and asked for informed consent ("deferred informed proxy consent") by the investigator or the treating physician within $72 \mathrm{~h}$ after ICU admission. If informed consent cannot be obtained within $72 \mathrm{~h}$, the patient will be excluded, and data will no longer be used. If the patient dies within $72 \mathrm{~h}$ after inclusion, prior to the informed consent procedure, the patient will not be excluded and data will be used [33]. The participant will be informed about participation in the study, if recovered. The patient is free to fill in a withdrawal form and

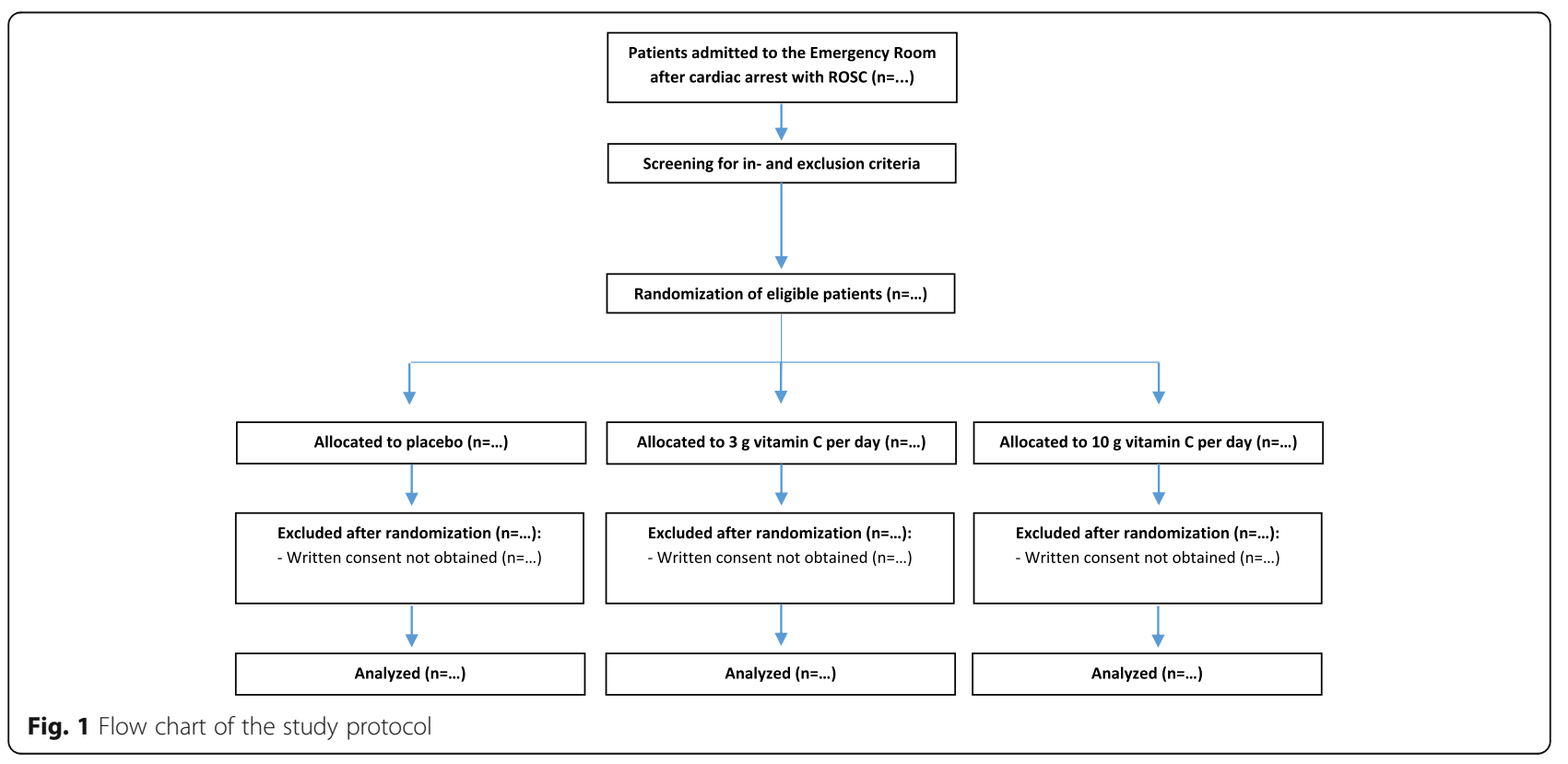


can decide to remove the collected data and body material.

\section{Data collection and outcome measures Data collection}

Data collection time points are presented in Fig. 2 (SPIRIT Figure). $\mathrm{T}_{0}$ is the time point of the first dose, as planned in a written or electronic order. The actual moment of administering the first dose of study medication may be earlier or delayed due to logistical processes at the Emergency Department, but is aimed to take place within $5 \mathrm{~h}$ after ROSC. The next doses are every $12 \mathrm{~h}$ after $\mathrm{T}_{0}$. Therefore, the time difference between $\mathrm{T}_{12}$ and $\mathrm{T}_{0}$ can be less than $12 \mathrm{~h}$.

To measure oxidative stress parameters and plasma vitamin $\mathrm{C}$ concentrations for pharmacokinetic modeling, the blood will be drawn daily in the coordinating center, before and directly after the infusion of study medication (baseline, trough, and peak plasma vitamin $C$ concentrations). In other participating hospitals, if logistically feasible, the blood will be drawn daily before the infusion of study medication (baseline and trough plasma vitamin $\mathrm{C}$ concentrations).

Day 1 is defined as the first $24 \mathrm{~h}$ after $\mathrm{T}_{0}$. Each next $24 \mathrm{~h}$ time period is defined as the following day: day 2 is $\mathrm{T}_{24}-\mathrm{T}_{48}$, day 3 is $\mathrm{T}_{48}-\mathrm{T}_{72}$, and day 4 is $\mathrm{T}_{72}-\mathrm{T}_{96}$. Followup telephone interviews and data collection will take place around day 28 and day 180. After pseudonymization, all data will be stored in Castor EDC (Electronic Data Capture), eCRF.

\section{Primary endpoint}

The primary endpoint is the delta $96 \mathrm{~h} R$-sequential organ failure assessment score $\left(\Delta \mathrm{R}-\mathrm{SOFA}_{96-\text { baseline}}\right)$ : The $\mathrm{R}$-SOFA score at $96 \mathrm{~h}$ after randomization minus the score after ROSC (baseline R-SOFA score). $\Delta \mathrm{R}$ -

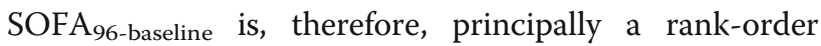
endpoint. After correction for baseline organ failure, each patient can fare better, worse, or equally well compared to another patient. SOFA scores are calculated according to the NICE criteria [34]. However, the SOFA score used in the present study is an adjusted SOFA score, the so-called Resuscitation SOFA (RSOFA), adjusted to assess the effect of the study intervention on organ dysfunction after cardiac arrest (see Additional File 1). In contrast to the classical SOFA score, for which worst values during a $24 \mathrm{~h}$ period are taken, the baseline and $\mathrm{T}_{96}$ SOFA scores are calculated within a shorter time window. The worst values during the first $24 \mathrm{~h}$ after ICU admission would not correctly reflect baseline organ dysfunction, as the study intervention which is administered early after ROSC could have influenced the degree of organ failure.

The adjustments are as follows:
1. Measurements must be reliable and should reflect the real organ function of the patient in a stable situation and not after an acute intervention.

a. Measurements should not be taken shortly after a bolus of catecholamines, propofol, or other sedatives, or after temporary ventilation with high oxygen fractions.

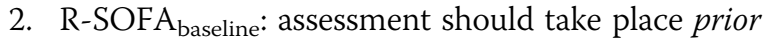
to the administration of the first study medication.

a. The first available bilirubin, platelet counts, and creatinine results will be taken, as these values do not change much during the first hours after hospital admission.

b. The last Glasgow Coma Scale (GCS) registered prior to the start of sedatives will be used.

c. Respiratory and/or hemodynamic parameters will be assessed in a time window of $2 \mathrm{~h}$ prior to the first dose. The worst validated parameters will be used.

i. If no validated data are available, the first available validated data point after the first dose will be used. It is allowed to extend the time window with a maximum of $2 \mathrm{~h}$ after the first dose has been administered.

3. R-SOFA 96 : assessment should take place within a comparable time window of $2 \mathrm{~h}\left(\mathrm{~T}_{95}-\mathrm{T}_{97}\right)$.

a. When bilirubin, platelet count and creatinine are not available within these $2 \mathrm{~h}$, the nearest value within $24 \mathrm{~h}$ prior to $\mathrm{T}_{96}$, and the nearest value within $24 \mathrm{~h}$ after $\mathrm{T}_{96}$ will be averaged.

b. The last GCS without sedation prior to $\mathrm{T}_{96}$ will be assessed.

c. The worst validated respiratory and/or hemodynamic parameters will be used.

i. If no validated data are available, the time window can be extended to $2 \mathrm{~h}$ before and 2 h after $\mathrm{T}_{96}\left(\mathrm{~T}_{94}-\mathrm{T}_{98}\right)$.

d. If a recovered patient is discharged from the ICU within $96 \mathrm{~h}$, the R-SOFA 96 will be the RSOFA score at ICU discharge.

e. Death at $96 \mathrm{~h}$ will be counted as the maximum R-SOFA score (24 points) as a worst-rank solution to informative censoring [35].

4. All SOFA components must be scored. If this is not possible based on the principles described above, these missing components will be discussed with an independent committee consisting of AdM, PE, and SR (coordinating investigators). They will decide which data point(s) can be used to score the organ(s), with our principles being the leading determinants.

\section{Secondary endpoints}

An overview of the secondary endpoints and data that will be collected is provided in Fig. 2 (SPIRIT Figure). 


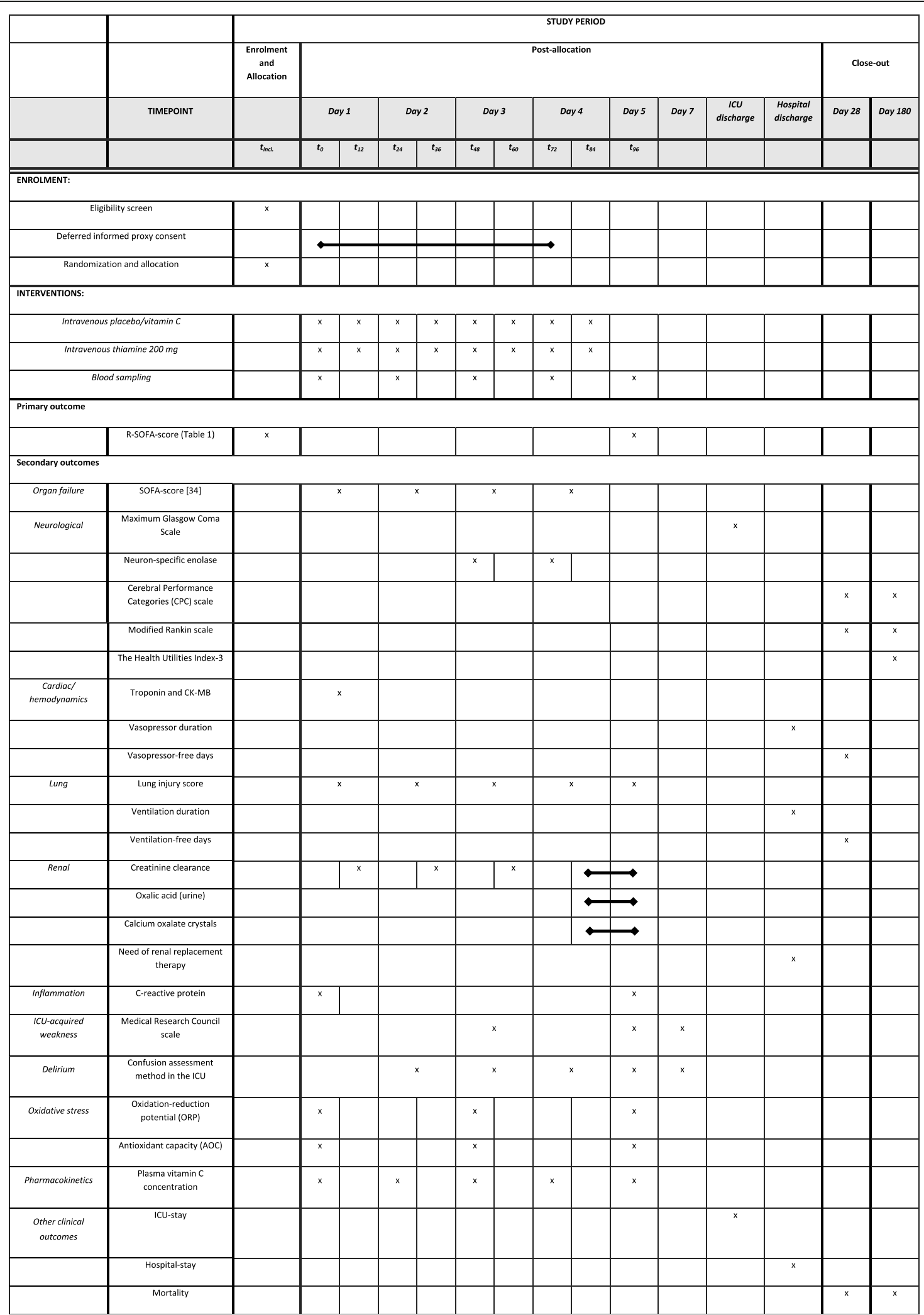

Fig. 2 Data collection and follow-up of the participants in the VITaCCA-trial (SPIRIT figure) 
An unadjusted SOFA-score [34] based on our 24 h time periods will be collected daily, as indicated in Fig. 2. The questionnaires will be carried out by telephone interviews [36]. In case a patient is not fully recovered and therefore not able to respond by phone, the questions will be asked to the legal representative of the patient.

Oxidative stress parameters will be measured using the RedoxSYS Diagnostic System (Aytu Bioscience, Englewood, CO, USA) [37]. In order to determine the plasma vitamin $\mathrm{C}$ concentration, blood samples will be processed immediately. After centrifugation, plasma is stabilized with $5.6 \%$ meta-phosphoric acid (1:5) to prevent oxidation of vitamin $\mathrm{C}$ and directly frozen at $-80{ }^{\circ} \mathrm{C}$ until vitamin $\mathrm{C}$ measurement.

\section{Other study parameters}

Demographics of the patient will be collected, including age, sex, body-mass index (weight/height ${ }^{2}$ ), any relevant medical history, and medication use. Furthermore, prehospital data regarding the resuscitation will be obtained from the ambulance service (i.e., witnessed arrest, bystander CPR, AED usage, number of shocks provided, first monitored rhythm, drugs given, used airway device, and total duration of resuscitation). All personal information of potential and enrolled participants will be handled and stored confidentially, according to the Dutch law and regulations.

\section{Statistical methods \\ Primary outcome}

The difference in $\triangle \mathrm{RSOFA}_{96}$-baseline score between intervention and control groups will be compared on a univariate basis (ANOVA with unadjusted analysis and after adjustment for, among others, age, site, prehospital parameters, and TTM) or (depending on normality) the Kruskal-Wallis test. In addition, both intervention groups will be combined and compared to placebo as well.

\section{Secondary outcomes}

Ordered categorical outcome variables (Glasgow Coma Score, Cerebral Performance Categories (CPC) scale, Modified Rankin scale (MRs), Health Utilities Index (HUI)) will be compared using the Cochran-Armitage test for trend. Continuous variables will be compared using linear mixed models or Friedman test as appropriate. Mortality will be analyzed using Kaplan-Meier curves and a Cox proportional hazards model with relevant prognostic covariates (especially time to return of spontaneous circulation). All statistical analyses will be performed according to the intention-to-treat principle. Moreover, the two vitamin $\mathrm{C}$ intervention groups will be combined for additional analyses.

\section{Sample size calculation}

$\triangle \mathrm{R}^{-} \mathrm{SOFA}_{96-\text { baseline }}$ has an estimated standard deviation of 3.5 points (highest estimate based on previous data in our hospital and a recent systematic review [38]). The study is powered to detect a clinically meaningful effect of 2.0 SOFA points difference between the trial arms. Eighty-two patients per group are needed to detect a treatment effect of 2 SOFA points with $90 \%$ power and a two-sided alpha of 0.01. The type-I error rate is set at 0.01 instead of 0.05 to minimize the risk of false-positive results. The most important reason for reducing the false-positive risk beyond the 0.05 norm is that there is a large worldwide interest in high-dose vitamin $C$ for critically ill patients. In this context, it was found important to reduce the fragility of the current trial in order to move beyond hypothesis-generating results. With an estimated $10 \%$ loss after randomization for late outcomes, the study aims to include $3 \times 90$ patients.

We consider a 2-point difference in $\Delta \mathrm{R}$ SOFA $_{96-\text { baseline }}$ between the intervention and control groups a meaningful effect. Firstly, a 2-point difference is sufficiently large to be clinically relevant and to impact future practice. For example, 2 SOFA points are the difference between mild neurologic deficit and coma, between moderate kidney dysfunction and dialysisdependent kidney failure, or between moderate oxygenation disturbance and life-threatening respiratory failure. Secondly, the association between $\triangle$ SOFA $_{96}$-baseline and outcomes is such that a 2-point difference is associated with measurable reductions in length-of-stay, mortality, and costs when the intervention is broadly implemented: each 1 point increase in $\triangle$ SOFA $_{96 \text {-baseline }}$ is associated with a $1.24(95 \%$ CI $1.04-1.47)$ odds ratio of mortality [4, 39].

\section{Safety}

The theoretical risks of intravenous supplementation of high-dose vitamin $\mathrm{C}$ are a paradoxical pro-oxidative effect in case of iron overload, oxalate kidney stones, and factitious hyperglycemia. First, literature shows that in vivo, the antioxidant effect of vitamin $\mathrm{C}$ predominates this indirect pro-oxidative effect, even in ironoverloaded human plasma [40]. As a precaution, patients with known hemochromatosis will be excluded from this study. Second, calcium oxalate stones and oxalate nephrocalcinosis take months and years to develop, respectively, and no studies with short-term vitamin $\mathrm{C}$ administration reported kidney stone formation [17-23, $25-28,41-48]$. To limit the conversion of vitamin $C$ to oxalate, all patients will receive thiamine $200 \mathrm{mg}$ every $12 \mathrm{~h}$ for 4 days [19]. Third, high-dose IV vitamin $\mathrm{C}$ can lead to factitious hyperglycemia when measured with point-of-care devices [49], mostly at dosages much higher than used in our study. We will measure blood 
glucose during the period of vitamin $\mathrm{C}$ administration by blood gas analysis or in the central laboratory.

Case reports suggest that large dosages $(>60 \mathrm{~g})$ of IV vitamin $\mathrm{C}$ may induce hemolysis in patients with G6PD deficiency. However, at a low-moderate dose, vitamin $\mathrm{C}$ supplementation may instead be the recommended treatment of drug-induced G6PD deficiency and should therefore not be considered contraindicated [50]. Nonetheless, patients with known G6PD deficiency will be excluded in this present trial.

\section{Adverse events, serious adverse events, and suspected unexpected serious adverse reactions}

Adverse events ("AEs") will not be reported. Every 3 months the Principal Investigator shall send a linelisting of all serious adverse events ("SAEs") to the Ethical Board of the coordinating center. For included patients, SAE's will be collected up to 28 days after inclusion. For excluded patients, only the total time of participating in the study will be used. Suspected unexpected serious adverse reaction ("SUSAR") will be reported immediately in accordance with the Dutch Medical Research Involving Human Subjects Act. Ancillary and post-trial care is not planned. An insurance has been taken out for everyone participating in this study. More insurance information can be found in the appendix of the information letter, which is available upon reasonable request.

\section{Data safety monitoring board and interim analysis}

An independent data safety monitoring board (DSMB) consisting of three independent members (a biostatistician, an intensivist, and a cardiologist) will evaluate safety during the trial. A blinded interim analysis focused on mortality differences will be carried out by an independent methodologist after the inclusion of 135 evaluable patients. The DSMB will be blinded but may be unblinded by the independent methodologist on request without any further clarification. In case of significantly increased mortality in the vitamin $\mathrm{C}$ group(s) compared to placebo-and after a thorough examination of the clinical content-the DSMB can advise to stop the further recruitment. The DSMB will assess the unblinded data before arriving at a material recommendation.

\section{Monitoring}

An independent monitor (quality officer) will monitor the study procedures and data quality according to the regulations described under Good Clinical Practice (GCP) in all participating centers. During onsite monitoring, the officer will perform source data verification of data in the Case Report Forms. In particular, adherence to inclusion and exclusion criteria, the deferred consent procedure, the primary endpoint, and the reporting of SAEs and SUSARs are subject to monitoring.

\section{Publication of trial results}

The trial results will be reported in accordance with the Consolidated Standards of Reporting Trials [51].

\section{SPIRIT Checklist}

The trial design follows the Standard Protocol Items Recommendations for Interventional Trials (SPIRIT). The SPIRIT Checklist is presented in Additional file 2.

\section{Discussion}

VITaCCA is the first double-blind, multi-center, randomized placebo-controlled trial to compare the effects of early high-dose vitamin $\mathrm{C}$ on organ dysfunction in patients after cardiac arrest.

The optimal dosing for intravenous vitamin $\mathrm{C}$ in the context of critical illness is still unknown and may depend on the patients' comorbid condition, on the severity of the acute disease, and on the subsequent degree of oxidative stress. We chose two different dose regimens in this study in order to observe differences in effect size between a supplementation and a pharmacological dose regimen. Our previous pharmacokinetic study demonstrates that $2 \mathrm{~g}$ a day was needed to restore plasma concentrations and that $10 \mathrm{~g}$ a day resulted in supranormal plasma concentrations [12]. This is in line with previous studies that show that $3 \mathrm{~g}$ a day was needed to restore deficient plasma concentrations [13]. Therefore, $3 \mathrm{~g}$ is chosen as a first dose regimen. To achieve supraphysiological plasma concentrations, $10 \mathrm{~g}$ will be used as a second dose regimen. This dose is in between $6 \mathrm{~g}$ and $16 \mathrm{~g}$ vitamin $\mathrm{C}$ a day, as used in previous studies [17-23, 2528, 44-46]. Currently, the optimal length of vitamin C supplementation is also unknown. We chose for 4 days, which is in line with previous studies in sepsis [17-23, $25,26,44,45]$. After these 4 days, vitamin $C$ administration will be lowered according to local guidelines, to allow the physiological signaling and repair function of low concentrations of ROS.

Delta SOFA score is chosen as the primary outcome since a recent meta-analysis demonstrates a strong reliable association with mortality, in comparison to a fixed day SOFA score [38]. Furthermore, a power calculation on a patient-oriented outcome such as mortality showed an unachievable required sample size of 3000 patients. The adjusted SOFA-score, the Resuscitation SOFA-score (R-SOFA), is designed to reliably assess baseline organ failure of post-cardiac arrest patients.

\section{Limitation}

We intended to use ready-to-use study medication produced by a pharmacy, but during the storage period, 
many (unknown) degradation products were formed, possibly due to the air permeability of the polypropylene vials. Therefore, we opted for a fresh preparation of the study medication as described in this protocol. A separate study is being conducted to identify and quantify the degradation products in different vitamin $\mathrm{C}$ solutions.

In conclusion, this study investigates the effect of early high-dose vitamin $C$ in post-cardiac arrest patients on organ dysfunction. If that proofs to be the case, intravenous vitamin $\mathrm{C}$ administration can be considered as a novel treatment for post-cardiac arrest patients.

\section{Trial status}

The first patient was included on October 20, 2019. The current version of the study protocol is version 9 , dated March 17, 2021. The estimated study completion date is January 2023.

\section{Abbreviations \\ AE: Adverse event; AED: Automatic external defibrillator; AOC: Antioxidant capacity; ANOVA: Analysis of variance; CPC: Cerebral performance categories; CPR: Cardiopulmonary resuscitation; DSMB: Data safety monitoring board; GCS: Glasgow coma scale; GCP: Good clinical practice; HUI: Health utilities index; ICU: Intensive care unit; IV: Intravenous; MRs: Modified ranking scale; NICE: Nationale Intensive Care Evaluatie; OHCA: Out-of-hospital cardiac arrest; ORP: Oxidation-reduction potential; PCl: Percutaneous coronary intervention; ROS: Reactive oxygen species; RRT: Renal replacement therapy; R- SOFA: Resuscitation-sequential organ failure assessment; ROSC: Return of spontaneous circulation; SAE: Serious adverse event; SUSAR: Suspected unexpected serious adverse reaction; TTM: Targeted temperature management; VF: Ventricular fibrillation; VT: Ventricular tachycardia; VITaCCA: Vitamin C in post-cardiac arrest syndrome}

\section{Supplementary Information}

The online version contains supplementary material available at https://doi. org/10.1186/s13063-021-05483-3.

Additional file 1:. Supplementary Table 1. Resuscitation Sequential Organ Failure Assessment Score (R-SOFA)

Additional file 2:. SPIRIT 2013 Checklist: Recommended items to address in a clinical trial protocol and related documents

\section{Acknowledgements}

We would like to express our thanks to the ARREST registry (Amsterdam Resuscitation studies), RAV Rotterdam-Rijnmond, RAV Gooi \& Vechtstreek, and RAV Gelderland-Midden for providing us prehospital patient data.

\section{Authors' contributions}

SR, HJdG, RB, AG, AdM, PE, and HOvS designed the study. SR is the coordinating investigator, and $A d M$ is the principal investigator in the coordinating center. CdU, ED, EJW, TR, AvZ, RV, and BvdB are the principal investigators of the participating hospitals. Authorship eligibility follows accepted academic standards. The authors read and approved the final manuscript.

\section{Funding}

Funding for the VITaCCA-trial was provided by contract to Amsterdam UMC, location VUmc, the study sponsor, by ZonMW (No 848081001). ZonMW will not have any input on the writing and submission of the manuscript, nor will they have any input on the writing of future manuscripts or decisions to publish data acquired in relation to this study.
Availability of data and materials

The datasets will be available from the corresponding author on reasonable request.

\section{Declarations}

\section{Ethics approval and consent to participate}

The study was approved by the ethics committee at Amsterdam UMC location VUmc (VU University Medical Centre Protocol Record METC2018.120). The ethics committee approved to start with study interventions before informed consent is obtained to avoid treatment delay. The legal representative will be informed and asked for informed consent ("deferred informed proxy consent") by the investigator or the treating physician within $72 \mathrm{~h}$ after ICU admission. If informed consent cannot be obtained within 72 $h$, the patient will be excluded, and data will no longer be used. The participant will be, if recovered, informed about participation in the study. The patient is free to fill in a withdrawal form and can decide to remove the collected data and body material. All substantial protocol amendments will be submitted to the Central Committee on Research Involving Human Subjects and the Medical Ethics Committee of Amsterdam UMC, location VUmc. Approved amendments will be communicated with the principal investigators of the participating hospitals.

\section{Consent for publication}

Not applicable.

\section{Competing interests}

The authors declare that they have no competing interests.

\section{Author details}

${ }^{1}$ Department of Intensive Care Medicine, Research VUmc Intensive Care (REVIVE), Amsterdam Cardiovascular Science (ACS), Amsterdam Infection and Immunity Institute (AI\&II), Amsterdam Medical Data Science (AMDS),

Amsterdam UMC, Location VUmc, Vrije Universiteit Amsterdam, De Boelelaan 1117, 1081 HV Amsterdam, The Netherlands. 'Department of Anesthesiology, Amsterdam UMC, Location VUmc, Vrije Universiteit Amsterdam, De Boelelaan 1117, 1081 HV Amsterdam, The Netherlands. ${ }^{3}$ Department of Intensive Care Medicine, Maasstad Hospital, Maasstadweg 21, 3079 DZ Rotterdam, The Netherlands. ${ }^{4}$ Department of Cardiology, Erasmus University Medical Center, Dr. Molewaterplein 40, 3015 GD Rotterdam, The Netherlands. ${ }^{5}$ Department of Intensive Care Medicine, Erasmus University Medical Center, Dr. Molewaterplein 40, 3015 GD Rotterdam, The Netherlands. ${ }^{6}$ Department of Intensive Care Medicine, Franciscus Gasthuis \& Vlietland, Kleiweg 500, 3045 PM Rotterdam, The Netherlands. ${ }^{7}$ Department of Anesthesiology, Intensive Care and Pain Medicine, Amphia Hospital, Molengracht 21, 4818 CK Breda, The Netherlands. ${ }^{8}$ Department of Intensive Care Medicine, Gelderse Vallei Hospital, Willy Brandtlaan 10, 6716 RP Ede, The Netherlands. ${ }^{9}$ Division of Human Nutrition and Health, Wageningen University \& Research, HELIX (Building 124), Stippeneng 4, 6708 WE Wageningen, The Netherlands. ${ }^{10}$ Department of Intensive Care Medicine, Tergooi Hospital, Van Riebeeckweg 212, 1213 XZ Hilversum, The Netherlands. ${ }^{11}$ Department of Intensive Care Medicine, OLVG, Oosterpark 9, 1091 AC Amsterdam, The Netherlands.

Received: 12 April 2021 Accepted: 23 July 2021

Published online: 18 August 2021

\section{References}

1. Stub D, Bernard S, Duffy SJ, Kaye DM. Post cardiac arrest syndrome: a review of therapeutic strategies. Circulation. 2011;123(13):1428-35. https://doi.org/1 $0.1161 /$ CIRCULATIONAHA.110.988725.

2. Spoelstra-de Man AME, Elbers PWG, Oudemans-van Straaten HM. Making sense of early high-dose intravenous vitamin $C$ in ischemia/reperfusion injury. Crit Care. 2018;22(1):70. https://doi.org/10.1186/s13054-018-1996-y.

3. Oudemans-van Straaten HM, Spoelstra-de Man AM, de Waard MC. Vitamin C revisited. Crit Care. 2014;18(4):460. https://doi.org/10.1186/s13054-014-04 $60-x$.

4. Grooth HJ, Spoelstra-de Man AME, Oudemans-van Straaten HM. Early plasma Vitamin C concentration, organ dysfunction and ICU mortality. Intensive Care Med. 2014;40(10 (Suppl 1)):S199.

5. Rozemeijer S, Spoelstra-de Man AME, Coenen S, Smit B, Elbers PWG, de Grooth $\mathrm{H}-$ J, et al. Estimating vitamin $\mathrm{C}$ status in critically ill patients with a 
novel point-of-care oxidation-reduction potential measurement. Nutrients. 2019;11(5):1031. https://doi.org/10.3390/nu11051031.

6. Gardner R, Liu X, Wang Y, Cole A, Heydrick S, Donnino MW, et al. Vitamin C levels amongst initial survivors of out of hospital cardiac arrest. Resuscitation. 2020;156:190-3. https://doi.org/10.1016/j.resuscitation.2020.09.005.

7. van Zanten AR, Sztark F, Kaisers UX, Zielmann S, Felbinger TW, Sablotzki AR, et al. High-protein enteral nutrition enriched with immune-modulating nutrients vs standard high-protein enteral nutrition and nosocomial infections in the ICU: a randomized clinical trial. JAMA. 2014;312(5):514-24. https://doi.org/10.1001/jama.2014.7698.

8. Levine M, Padayatty SJ, Espey MG. Vitamin C: a concentration-function approach yields pharmacology and therapeutic discoveries. Adv Nutr. 2011; 2(2):78-88. https://doi.org/10.3945/an.110.000109.

9. Padayatty SJ, Sun H, Wang Y, Riordan HD, Hewitt SM, Katz A, et al. Vitamin C pharmacokinetics: implications for oral and intravenous use. Ann Intern Med. 2004;140(7):533-7. https://doi.org/10.7326/0003-4819-140-7-200404060-00010.

10. Carr AC, Rosengrave PC, Bayer S, Chambers S, Mehrtens J, Shaw GM Hypovitaminosis $C$ and vitamin C deficiency in critically ill patients despite recommended enteral and parenteral intakes. Crit Care. 2017;21(1):300. https://doi.org/10.1186/s13054-017-1891-y.

11. Spoelstra-de Man AME, Elbers PWG, Oudemans-Van Straaten HM. Vitamin C: should we supplement? Curr Opin Crit Care. 2018;24(4):248-55. https://doi. org/10.1097/MCC.0000000000000510.

12. de Grooth HJ, Manubulu-Choo WP, Zandvliet AS, Spoelstra-de Man AME, Girbes AR, Swart EL, et al. Vitamin C pharmacokinetics in critically ill patients: a randomized trial of four IV regimens. Chest. 2018;153(6):1368-77. https:// doi.org/10.1016/j.chest.2018.02.025.

13. Long CL, Maull Kl, Krishnan RS, Laws HL, Geiger JW, Borghesi L, et al. Ascorbic acid dynamics in the seriously ill and injured. J Surg Res. 2003; 109(2):144-8. https://doi.org/10.1016/S0022-4804(02)00083-5.

14. Hudson EP, Collie JT, Fujii T, Luethi N, Udy AA, Doherty S, et al. Pharmacokinetic data support 6-hourly dosing of intravenous vitamin C to critically ill patients with septic shock. Crit Care Resusc. 2019;21(4):236-42.

15. Khan SA, Bhattacharjee S, Ghani MOA, Walden R, Chen QM. Vitamin C for cardiac protection during percutaneous coronary intervention: a systematic review of randomized controlled trials. Nutrients. 2020;12(8).

16. Hill A, Clasen KC, Wendt S, Majoros AG, Stoppe C, Adhikari NKJ, et al. Effects of vitamin $C$ on organ function in cardiac surgery patients: a systematic review and meta-analysis. Nutrients. 2019;11(9).

17. Fowler AA III, Syed AA, Knowlson S, Sculthorpe R, Farthing D, DeWilde C, et al. Phase I safety trial of intravenous ascorbic acid in patients with severe sepsis. J Trans/ Med. 2014;12(1):32. https://doi.org/10.1186/1479-5876-12-32.

18. Zabet MH, Mohammadi M, Ramezani M, Khalili H. Effect of high-dose ascorbic acid on vasopressor's requirement in septic shock. J Res Pharm Pract. 2016;5(2):94-100.

19. Marik PE, Khangoora V, Rivera R, Hooper MH, Catravas J. Hydrocortisone, vitamin $C$, and thiamine for the treatment of severe sepsis and septic shock: a retrospective before-after study. Chest. 2017;151(6):1229-38. https://doi. org/10.1016/j.chest.2016.11.036

20. Fowler AA 3rd, Truwit JD, Hite RD, Morris PE, DeWilde C, Priday A, et al. Effect of vitamin $C$ infusion on organ failure and biomarkers of inflammation and vascular injury in patients with sepsis and severe acute respiratory failure: the CITRIS-ALI randomized clinical trial. JAMA. 2019; 322(13):1261-70. https://doi.org/10.1001/jama.2019.11825.

21. Moskowitz A, Huang DT, Hou PC, Gong J, Doshi PB, Grossestreuer AV, et al. Effect of ascorbic acid, corticosteroids, and thiamine on organ injury in septic shock: the ACTS randomized clinical trial. JAMA. 2020;324(7):642-50. https://doi.org/10.1001/jama.2020.11946.

22. Iglesias J, Vassallo AV, Patel W, Sullivan JB, Cavanaugh J, Elbaga Y. Outcomes of metabolic resuscitation using ascorbic acid, thiamine, and glucocorticoids in the early treatment of sepsis: the ORANGES trial. Chest. 2020;158(1):164-73. https://doi.org/10.1016/j.chest.2020.02.049.

23. Chang P, Liao Y, Guan J, Guo Y, Zhao M, Hu J, et al. Combined treatment with hydrocortisone, vitamin $C$, and thiamine for sepsis and septic shock: a randomized controlled trial. Chest. 2020;158(1):174-82. https://doi.org/10.101 6/j.chest.2020.02.065

24. Fujii T, Udy AA. Additional trials of vitamin C in septic shock: a bag of mixed fruit. Chest. 2020;158(1):13-4. https://doi.org/10.1016/j.chest.2020. 03.030 .

25. Mohamed ZU, Prasannan P, Moni M, Edathadathil F, Prasanna P, Menon A, et al. Vitamin C therapy for routine care in septic shock (ViCTOR) trial: effect of intravenous vitamin C, thiamine, and hydrocortisone administration on inpatient mortality among patients with septic shock. Indian J Crit Care Med. 2020;24(8):653-61.

26. Wani SJ, Mufti SA, Jan RA, Shah SU, Qadri SM, Khan UH, et al. Combination of vitamin C, thiamine and hydrocortisone added to standard treatment in the management of sepsis: results from an open label randomised controlled clinical trial and a review of the literature. Infect Dis Ther. 2020; 52(4):271-8. https://doi.org/10.1080/23744235.2020.1718200.

27. Hwang SY, Ryoo SM, Park JE, Jo YH, Jang D-H, Suh GJ, et al. Combination therapy of vitamin C and thiamine for septic shock: a multi-centre, doubleblinded randomized, controlled study. Intensive Care Med. 2020;46(11):201525. https://doi.org/10.1007/s00134-020-06191-3.

28. Fujii T, Luethi N, Young PJ, Frei DR, Eastwood GM, French CJ, et al. Effect of vitamin C, hydrocortisone, and thiamine vs hydrocortisone alone on time alive and free of vasopressor support among patients with septic shock: the VITAMINS randomized clinical trial. JAMA. 2020;323(5):423-31. https://doi. org/10.1001/jama.2019.22176.

29. Fujii T, Fowler R, Vincent J-L. Vitamin C and thiamine for sepsis: time to go back to fundamental principles. Intensive Care Med. 2020;46(11):2061-3. https://doi.org/10.1007/s00134-020-06242-9.

30. Beesems JA, Stieglis R, Koster RW. Reanimatie buiten het ziekenhuis in Noord-Holland en twente: resultaten ARREST-onderzoek 2006-2011. 2012.

31. Nolan JP, Soar J, Cariou A, Cronberg T, Moulaert VR, Deakin CD, et al. European Resuscitation Council and European Society of Intensive Care Medicine 2015 guidelines for post-resuscitation care. Intensive Care Med. 2015;41(12):2039-56. https://doi.org/10.1007/s00134-015-4051-3.

32. Richtlijn Prognose van postanoxisch coma. https://richtlijnendatabase.nl/ richtlijn/prognose_van_postanoxisch_coma/startpagina.html.

33. Flowcharts deferred consent for medical research in emergency situations. https://english.ccmo.nl/latest/news/2020/05/12/flowcharts-deferred-consentfor-medical-research-in-emergency-situations.

34. Sequential Organ Failure Assessment (SOFA). https://www.stichting-nice.nl/ download/?Groep=Data\%20Dictionary\&Taal=Engels.

35. Lachin JM. Worst-rank score analysis with informatively missing observations in clinical trials. Control Clin Trials. 1999;20(5):408-22. https://doi.org/10.1016/ S0197-2456(99)00022-7.

36. Haywood K, Whitehead L, Nadkarni VM, Achana F, Beesems S, Bottiger BW, et al. COSCA (Core Outcome Set for Cardiac Arrest) in adults: an advisory statement from the International Liaison Committee on Resuscitation. Circulation. 2018;137(22):e783-801.

37. Rael LT, Bar-Or R, Kelly MT, Carrick MM, Bar-Or D. Assessment of oxidative stress in patients with an isolated traumatic brain injury using disposable electrochemical test strips. Electroanalysis. 2015;27:2567-73.

38. de Grooth HJ, Geenen IL, Girbes AR, Vincent JL, Parienti JJ, Oudemans-van Straaten HM. SOFA and mortality endpoints in randomized controlled trials: a systematic review and meta-regression analysis. Crit Care. 2017;21(1):38. https://doi.org/10.1186/s13054-017-1609-1.

39. Ferreira FL, Bota DP, Bross A, Melot C, Vincent JL. Serial evaluation of the SOFA score to predict outcome in critically ill patients. JAMA. 2001;286(14): 1754-8. https://doi.org/10.1001/jama.286.14.1754.

40. Carr A, Frei B. Does vitamin C act as a pro-oxidant under physiological conditions? FASEB J. 1999;13(9):1007-24. https://doi.org/10.1096/fasebj.13.9.1007.

41. Nathens AB, Neff MJ, Jurkovich GJ, Klotz P, Farver K, Ruzinski JT, et al. Randomized, prospective trial of antioxidant supplementation in critically ill surgical patients. Ann Surg. 2002:236(6):814-22. https://doi.org/10.1097/ 00000658-200212000-00014

42. Tanaka H, Matsuda T, Miyagantani Y, Yukioka T, Matsuda H, Shimazaki S. Reduction of resuscitation fluid volumes in severely burned patients using ascorbic acid administration: a randomized, prospective study. Arch Surg. 2000;135(3):326-31. https://doi.org/10.1001/archsurg.135.3.326.

43. Prier M, Carr AC, Baillie N. No reported renal stones with intravenous vitamin $C$ administration: a prospective case series study. Antioxidants (Basel). 2018;7(5).

44. Mitchell AB, Ryan TE, Gillion AR, Wells LD, Muthiah MP. Vitamin C and thiamine for sepsis and septic shock. Am J Med. 2020;133(5):635-8. https:// doi.org/10.1016/j.amjmed.2019.07.054.

45. Kim WY, Jo EJ, Eom JS, Mok J, Kim MH, Kim KU, et al. Combined vitamin C, hydrocortisone, and thiamine therapy for patients with severe pneumonia who were admitted to the intensive care unit: propensity score-based analysis of a before-after cohort study. J Crit Care. 2018;47:211-8. https://doi. org/10.1016/j.jcrc.2018.07.004. 
46. Reddy PR, Samavedam S, Aluru N, Yelle S, Rajyalakshmi B. Metabolic resuscitation using hydrocortisone, ascorbic acid, and thiamine: do individual components influence reversal of shock independently? Indian J Crit Care Med. 2020;24(8):649-52. https://doi.org/10.5005/jp-journals-1 0071-23515.

47. Wald EL, Sanchez-Pinto LN, Smith CM, Moran T, Badke CM, Barhight MF, et al. Hydrocortisone-ascorbic acid-thiamine use associated with lower mortality in pediatric septic shock. Am J Respir Crit Care Med. 2020;201(7): 863-7. https://doi.org/10.1164/rccm.201908-1543LE.

48. Nagel SS, Radu CA, Kremer T, Meess D, Horter J, Ziegler B, et al. Safety, pharmacodynamics, and efficacy of high- versus low-dose ascorbic acid in severely burned adults. Journal of Burn Care \& Research. 2020;41(4):871-7. https://doi.org/10.1093/jbcr/iraa041.

49. Cho J, Ahn S, Yim J, Cheon Y, Jeong SH, Lee SG, et al. Influence of vitamin $C$ and maltose on the accuracy of three models of glucose meters. Ann Lab Med. 2016;36(3):271-4. https://doi.org/10.3343/alm.2016.36.3.271.

50. Marik PE. Is intravenous vitamin C contraindicated in patients with G6PD deficiency? Crit Care. 2019;23(1):109. https://doi.org/10.1186/s13054-019-23 97-6.

51. Schulz KF, Altman DG, Moher D, Group C. CONSORT 2010 statement: updated guidelines for reporting parallel group randomised trials. BMJ. 2010;340(mar23 1):c332. https://doi.org/10.1136/bmj.c332.

\section{Publisher's Note}

Springer Nature remains neutral with regard to jurisdictional claims in published maps and institutional affiliations.

Ready to submit your research? Choose BMC and benefit from:

- fast, convenient online submission

- thorough peer review by experienced researchers in your field

- rapid publication on acceptance

- support for research data, including large and complex data types

- gold Open Access which fosters wider collaboration and increased citations

- maximum visibility for your research: over $100 \mathrm{M}$ website views per year

At $\mathrm{BMC}$, research is always in progress.

Learn more biomedcentral.com/submissions 